\title{
Analytic framework for the effective rate of MISO fading channels
}

Michail Matthaiou, George C. Alexandropoulos, Hien Quoc Ngo and Erik G. Larsson

\section{Linköping University Post Print}

N.B.: When citing this work, cite the original article.

(C2012 IEEE. Personal use of this material is permitted. However, permission to reprint/republish this material for advertising or promotional purposes or for creating new collective works for resale or redistribution to servers or lists, or to reuse any copyrighted component of this work in other works must be obtained from the IEEE.

Michail Matthaiou, George C. Alexandropoulos, Hien Quoc Ngo and Erik G. Larsson, Analytic framework for the effective rate of MISO fading channels, 2012, IEEE Transactions on Communications, (60), 6, 1741-1751. http://dx.doi.org/10.1109/TCOMM.2012.040212.110783

Postprint available at: Linköping University Electronic Press

http://urn.kb.se/resolve?urn=urn:nbn:se:liu:diva-74570 


\title{
Analytic Framework for the Effective Rate of MISO Fading Channels
}

\author{
Michail Matthaiou, Member, IEEE, George C. Alexandropoulos, Member, IEEE, \\ Hien Quoc Ngo, Student Member, IEEE, and Erik G. Larsson, Senior Member, IEEE
}

\begin{abstract}
The delay constraints imposed by future wireless applications require a suitable metric for assessing their impact on the overall system performance. Since the classical Shannon's ergodic capacity fails to do so, the so-called effective rate was recently established as a rigorous alternative. While prior relevant works have improved our knowledge on the effective rate characterization of communication systems, an analytical framework encompassing several fading models of interest is not yet available. In this paper, we pursue a detailed effective rate analysis of Nakagami- $m$, Rician and generalized- $\mathcal{K}$ multipleinput single-output (MISO) fading channels by deriving new, analytical expressions for their exact effective rate. Moreover, we consider the asymptotically low and high signal-to-noise regimes, for which tractable, closed-form effective rate expressions are presented. These results enable us to draw useful conclusions about the impact of system parameters on the effective rate of different MISO fading channels. All the theoretical expressions are validated via Monte-Carlo simulations.
\end{abstract}

Index Terms-Delay constraints, effective rate, fading channels, multiple-input single-output (MISO) systems.

\section{INTRODUCTION}

T HE capacity performance and limits of multiple-antenna technologies have been well investigated in the corresponding literature (see [1] and references therein among others). In this context, the typical metric for performance evaluation has been Shannon's ergodic capacity (or outage capacity for non-ergodic channels). However, some of the most important emerging applications (e.g., voice over IP (VoIP), interactive and multimedia streaming, interactive gaming, mobile TV and computing) impose stringent quality of service (QoS) constraints; such constraints typically appear in the

Paper approved by N. C. Beaulieu, the Editor for Wireless Communication Theory of the IEEE Communications Society. Manuscript received November 18, 2011; accepted January 23, 2012.

The work of M. Matthaiou has been supported in part by the Swedish Governmental Agency for Innovation Systems (VINNOVA) within the VINN Excellence Center Chase. The work of H. Q. Ngo and E. G. Larsson was supported in part by the Swedish Research Council (VR), the Swedish Foundation for Strategic Research (SSF), and ELLIIT. E. G. Larsson is a Royal Swedish Academy of Sciences (KVA) Research Fellow supported by a grant from the Knut and Alice Wallenberg Foundation. Part of the paper has been presented at the IEEE Sensor and Multichannel Signal Processing Workshop (SAM), Hoboken, NJ, June 2012.

M. Matthaiou is with the Department of Signals and Systems, Chalmers University of Technology, 412 96, Gothenburg, Sweden (e-mail: michail.matthaiou@chalmers.se).

G. C. Alexandropoulos is with the Athens Information Technology (AIT) 19.5 km Markopoulo Ave., 19002, Athens, Greece (e-mail: alexandg@ait.gr).

H. Q. Ngo and E. G. Larsson are with the Department of Electrica Engineering (ISY), Linköping University, 581 83, Linköping, Sweden (e-mail: \{nqhien, erik.larsson\}@isy.liu.se).

Digital Object Identifier 10.1109/TCOMM.2012.09.110783 form of constraints on queuing delays or queue lengths. These applications are inherently delay-sensitive, which implies that the data will expire if it is not successfully delivered within a time frame. As such, a QoS metric that is able to capture the delay constraints of communication systems becomes of vital importance. Unfortunately, the conventional notion of Shannon capacity cannot account for the delay aspect.

Motivated by these observations, the authors in [2] introduced effective capacity as an appropriate metric to quantify the system performance under QoS limitations, such as data rate, delay and delay-violation probability. Since then, this area has attracted considerable research interest following the need of next-generation wireless systems to support diverse QoS requirements and traffic characteristics. More particularly, we first note the works [3]-[7] which explored the effective capacity of different single-antenna communication systems. A plethora of recent works focused on the effective capacity of multiple-antenna communication systems. In this context, [8] investigated the effective capacity of Gaussian quasi-static block-fading multiple-input multiple-output (MIMO) systems in independent and identically distributed (i.i.d.) Rayleigh fading channels. Moreover, [9] derived the optimal precoding scheme with covariance feedback for correlated multiple-input single-output (MISO) systems. Recently, [10] examined in detail the MIMO effective capacity in the high and low signalto-noise (SNR) regimes and demonstrated the interactions between the queuing constraints and spatial dimensions over a wide range of SNR values. Finally, [11] considered the effective capacity of MISO systems by taking into account the effects of spatial correlation. By doing so, it was theoretically shown, using principles of majorization theory, that correlation always reduces effective capacity.

The common characteristic of the above mentioned works [8]-[11], however, is that they adopt the assumption of Rayleigh fading. Although the assumption of Rayleigh fading simplifies extensively the performance analysis of multipleantenna systems, its validity is often violated in practical propagation scenarios [12]. Yet, very little is still known about the effective capacity of multiple-antenna systems in nonRayleigh fading conditions. In this light, we herein bridge this gap by analytically investigating the effective rate of MISO systems for several more general fading models. In particular, the contributions of this paper can be summarized as:

- We elaborate on three popular channel fading models, namely Nakagami- $m$, Rician, and generalized- $\mathcal{K}$ models, which have been extensively used for the performance 
analysis of wireless communication systems [12]. For these fading models, new analytical expressions for the exact effective rate are derived. For the particular case of Nakagami- $m$ fading, two novel upper bounds on the effective rate are also proposed. Note that, although the considered models incur significant mathematical challenges, all the presented results can be easily evaluated.

- In order to get additional insights into the impact of system parameters, such as delay constraints, fading parameters and number of antennas, we consider the asymptotically low and high-SNR regimes. In these asymptotic cases, we investigate the notions of minimum normalized energy per information bit to reliably convey any positive rate and wideband slope, along with the high-SNR slope and high-SNR power offset, respectively. For these metrics, new tractable expressions are deduced that extend and complement previous results on Rayleigh fading channels. For the sake of completeness, the link of the presented results with previously reported results is also provided.

The rest of the paper is organized as follows: In Section II, the MISO channel model is introduced along with the concepts of effective capacity and effective rate. In Section III, we pursue a detailed effective rate analysis of several fading channel models. A set of numerical results is also provided to validate the theoretical analysis. Finally, Section IV concludes the paper and summarizes the key findings.

Notation: We use upper and lower case boldface to denote matrices and vectors, respectively. The symbol $(\cdot)^{\dagger}$ represents the Hermitian transpose, while $\operatorname{tr}(\cdot)$ yields the matrix trace. The expectation of a random variable is denoted as $\mathrm{E}\{\cdot\}$, and $\operatorname{Pr}(\cdot)$ represents probability. The symbol $\stackrel{a . s .}{\rightarrow}$ denotes almost sure convergence.

\section{SYSTEM MODEL}

We consider a MISO system with $N_{t}$ transmit antennas whose complex input-output relationship can be expressed as

$$
y=\mathbf{h x}+n
$$

where $\mathbf{h} \in \mathbb{C}^{1 \times N_{t}}$ represents the MISO channel fading vector, while $\mathbf{x} \in \mathbb{C}^{N_{t} \times 1}$ and $n$ denote the transmitted vector and the complex additive white Gaussian noise (AWGN) term with zero-mean and variance $N_{0}$, respectively. According to [2], the effective capacity is defined as the maximum constant arrival rate that a given service process can support in order to guarantee a statistical QoS requirement, specified by the QoS exponent $\theta$. Assuming block fading channels, the effective capacity is defined as [8]

$$
a(\theta)=-\frac{1}{\theta T} \ln \{\mathrm{E}\{\exp (-\theta T C)\}\}, \theta \neq 0
$$

where $T$ is the block-length, $C$ is the transmission rate which is a random variable (RV), and the expectation is taken over $C$. It is noted that the parameter $\theta$ determines to so-called asymptotic decay-rate of the buffer occupancy and is given by

$$
\theta=-\lim _{x \rightarrow \infty} \frac{\ln \operatorname{Pr}[L>x]}{x}
$$

where $L$ is the equilibrium queue-length of the buffer at the transmitter [2]. Then, assuming that the transmitter sends uncorrelated circularly symmetric zero-mean complex Gaussian signals and uniform power allocation across the transmit antennas, the effective rate can be succinctly expressed as follows ${ }^{1}$

$$
\mathcal{R}(\rho, \theta)=-\frac{1}{A} \log _{2}\left(\mathrm{E}\left\{\left(1+\frac{\rho}{N_{t}} \mathbf{h h}^{\dagger}\right)^{-A}\right\}\right) \quad \text { bits/s/Hz }
$$

where $A \triangleq \theta T B / \ln 2$, with $B$ denoting the bandwidth of the system, while $\rho$ is the average transmit SNR. Evidently, for $\theta=0$, i.e., no delay constraints, the effective capacity coincides with the well established concept of ergodic rate of the corresponding wireless channel.

\section{EFFECTIVE RATE ANALysis of FADing Channels}

In this section, we present a detailed effective rate analysis of three popular fading channel models, namely Nakagami- $m$, Rician and generalized- $\mathcal{K}$, assuming i.i.d. fading across the transmit antennas. Note that the scenarios of non-identically distributed or correlated fading can be addressed using the same methodologies as in the following. Such an analysis remains an important topic for further work, though we do not pursue it further in this paper.

\section{A. Nakagami-m fading channels}

The Nakagami- $m$ distribution, where $m$ is the Nakagami$m$ factor, is a general fading model that includes the onesided Gaussian distribution (for $m=1 / 2$ ) and the Rayleigh distribution $(m=1)$ as special cases [13]. It has been demonstrated that it often yields good fit with measured data in various land-mobile [14] and indoor-mobile multipath propagation environments [15], [16]. In this case, the entries of the channel vector $\mathbf{h}$ are i.i.d. Nakagami-m RVs with parameters $m$ and $\Omega$, where $\Omega$ is the average fading power. Then, the probability density function (p.d.f.) of $x=\left|h_{k}\right|^{2}$ $\left(k=1, \ldots, N_{t}\right)$, where $h_{k}$ is the $k$-th entry of $\mathbf{h}$, is given by

$$
p(x)=\frac{x^{m-1}}{\Gamma(m)}\left(\frac{m}{\Omega}\right)^{m} \exp \left(-\frac{m}{\Omega} x\right), \quad m \geq 0.5, \Omega \geq 0
$$

where $\Gamma(x)=\int_{0}^{\infty} t^{x-1} \exp (-t) d t$ represents the Gamma function [17, Eq. (8.310.1)].

1) Exact analysis: We first consider the exact effective rate $\mathcal{R}(\rho, \theta)$ as follows:

Proposition 1: For Nakagami- $m$ fading, the effective rate of MISO channels is given by

$$
\begin{aligned}
\mathcal{R}(\rho, \theta) & =\frac{m N_{t}}{A} \log _{2}\left(\frac{\Omega \rho}{m N_{t}}\right) \\
& -\frac{1}{A} \log _{2}\left(U\left(m N_{t} ; m N_{t}+1-A ; \frac{m N_{t}}{\Omega \rho}\right)\right)
\end{aligned}
$$

\footnotetext{
${ }^{1}$ Henceforth, we will be using the terminology effective rate instead of effective capacity, since we perform no optimization over the input covariance matrix $\mathbf{Q}=\mathbf{E}\left\{\mathbf{x x}^{\dagger}\right\}$. For a detailed discussion, interested readers are referred to [10].
} 


$$
=\log _{2}\left(\frac{\Omega \rho}{m N_{t}}\right)-\frac{1}{A} \log _{2}\left(U\left(A ; A+1-m N_{t} ; \frac{m N_{t}}{\Omega \rho}\right)\right)
$$

where $U(\cdot)$ is the Tricomi hypergeometric function [18, Eq. (13.1.3)].

Proof: In order to evaluate the expectation in (4), we first need to determine the p.d.f. of the sum of $N_{t}$ i.i.d. Gamma $\mathrm{RVs}$, defined as $z=\sum_{k=1}^{N_{t}}\left|h_{k}\right|^{2}$. In general, it is known (see e.g., [19]) that the sum of $n$ statistically independent Gamma RVs with shape parameters $\left\{c_{i}\right\}_{i=1}^{n}$ and a common scale parameter $b$, is also a gamma RV with parameters $\sum_{k=1}^{n} c_{i}$ and $b$. As such, we can easily obtain for the p.d.f. of $z$,

$$
p(z)=\frac{z^{m N_{t}-1}}{\Gamma\left(m N_{t}\right)}\left(\frac{m}{\Omega}\right)^{m N_{t}} \exp \left(-\frac{m z}{\Omega}\right) .
$$

Substituting (8) into (4), we can obtain the desired result in (6) after evaluating the involved integral with the help of the following identity [20, Eq. (39)]

$$
\int_{0}^{\infty}(1+a x)^{-\nu} x^{q-1} e^{-p x} d x=\frac{\Gamma(q)}{a^{q}} U\left(q ; q+1-\nu ; \frac{p}{a}\right) .
$$

The proof concludes by recalling Kummer's transformation $U(a ; b ; x)=x^{1-b} U(a-b+1 ; 2-b ; x)$ [21, Eq. (07.33.17.0007.01)] to obtain (7).

In addition to the exact results given by Proposition 1, we now propose two new analytical upper bounds on the effective rate of Nakagami- $m$ fading channels.

Theorem 1: For Nakagami- $m$ fading, the effective rate of MISO channels is upper bounded as $\mathcal{R}(\rho, \theta) \leq \mathcal{R}_{u 1} \leq \mathcal{R}_{u 2}$, with

$$
\mathcal{R}_{u 1}=\left\{\begin{array}{l}
\frac{\Omega \rho}{\ln 2}{ }_{3} F_{1}\left(1+m N_{t}, 1,1 ; 2,-\frac{\Omega \rho}{m N_{t}}\right) \\
\frac{1}{\ln 2} \exp \left(\frac{m N_{t}}{\Omega \rho}\right) \sum_{k=1}^{m N_{t}} E_{k}\left(\frac{m N_{t}}{\Omega \rho}\right), m N_{t} \in \mathbb{Z}^{+}
\end{array}\right.
$$

$$
\mathcal{R}_{u 2}=\log _{2}(1+\Omega \rho)
$$

where ${ }_{p} F_{q}(\cdot)$ represents the generalized hypergeometric function with $p, q \in \mathbb{Z}$ [17, Eq. (9.14.1)], while $E_{n}(x)=$ $\int_{1}^{\infty} \frac{e^{-x t}}{t^{n}} d t$ is the exponential integral function [18, Eq. (5.1.4)] of order $n$, for $n=0,1, \ldots$ and $\operatorname{Re}(x)>0$.

Proof: The proof is essentially an application of Jensen's inequality. In particular, we can first exploit the fact that $-\log _{2}(\cdot)$ is a $\log$-convex function to upper bound the effective rate in (4) according to

$$
\begin{aligned}
\mathcal{R}(\rho, \theta) & \leq-\frac{1}{A} \mathrm{E}\left\{\log _{2}\left(\left(1+\frac{\rho}{N_{t}} \mathbf{h} \mathbf{h}^{\dagger}\right)^{-A}\right)\right\} \\
& =\mathrm{E}\left\{\log _{2}\left(1+\frac{\rho}{N_{t}} \mathbf{h} \mathbf{h}^{\dagger}\right)\right\} \\
& \leq \log _{2}\left(1+\frac{\rho}{N_{t}} \mathrm{E}\left\{\mathbf{h} \mathbf{h}^{\dagger}\right\}\right)
\end{aligned}
$$

where (14) follows since $\log _{2}(\cdot)$ is a concave function. Note that from (13) and (14) we will respectively obtain $\mathcal{R}_{u 1}$ and $\mathcal{R}_{u 2}$. As such, we can express $\mathcal{R}_{u 1}, \mathcal{R}_{u 2}$ in the following

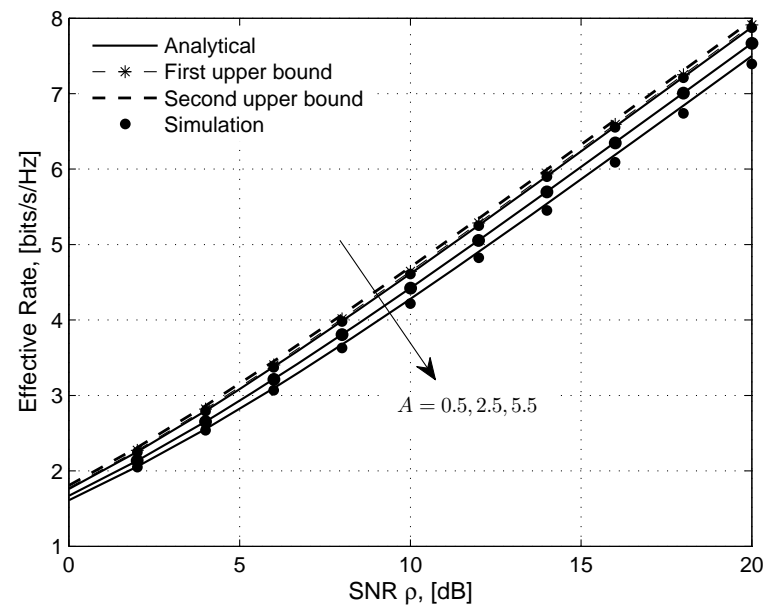

Fig. 1. Simulated effective rate, analytical expression and upper bounds against the SNR for Nakagami- $m$ fading $\left(N_{t}=6, m=2, \Omega=2.5\right)$.

integral form:

$$
\begin{aligned}
& \mathcal{R}_{u 1}=\frac{1}{\ln 2} \int_{0}^{\infty} \ln \left(1+\frac{\rho}{N_{t}} z\right) p(z) d z \\
& \mathcal{R}_{u 2}=\log _{2}\left(1+\frac{\rho}{N_{t}} \int_{0}^{\infty} z p(z) d z\right) .
\end{aligned}
$$

In order to evaluate the involved integrals, we employ some standard methodologies taken from [22] and [23], and thereafter simplify the results. By doing so, we have for $\mathcal{R}_{u 1}$

$$
\mathcal{R}_{u 1}=\frac{1}{\Gamma\left(m N_{t}\right) \ln 2} G_{3,2}^{1,3}\left[\frac{\Omega \rho}{m N_{t}} \mid \begin{array}{c}
1-m N_{t}, 1,1 \\
1,0
\end{array}\right]
$$

where $G_{p, q}^{r, s}\left[x, \mid \begin{array}{l}\alpha_{1}, \ldots, \alpha_{p} \\ \beta_{1}, \ldots, \beta_{q}\end{array}\right]$ denotes the Meijer's- $G$ function [17, Eq. (9.301)]. The above formula can be re-expressed with the aid of [17, Eq. (9.31.5)] according to

$$
G_{3,2}^{1,3}\left[\frac{\Omega \rho}{m N_{t}} \mid \begin{array}{c}
1-m N_{t}, 1,1 \\
1,0
\end{array}\right]=\frac{\Omega \rho}{m N_{t}} G_{3,2}^{1,3}\left[\frac{\Omega \rho}{m N_{t}} \mid \begin{array}{c}
-m N_{t}, 0,0 \\
0,-1
\end{array}\right] .
$$

Then, using [24, Eq. (8.4.51.1)] and the property $\Gamma(x+$ $1)=x \Gamma(x)$ [18, Eq. (6.1.15)], we can obtain the desired result, in terms of a hypergeometric function, after appropriate simplifications.

Clearly, $\mathcal{R}_{u 1}$ is harder to evaluate but is inherently tighter than $\mathcal{R}_{u 2}$. Both bounds, however, are independent of the delay constraints while $\mathcal{R}_{u 1}$ is identical to the ergodic capacity of a single-antenna fast-fading channel under Nakagami- $m$ fading conditions. We note that when the number of transmit antennas $N_{t}$ grows large, both bounds become exact and equal to $\mathcal{R}_{u 2}$. This is due to the law of large numbers which states that $\frac{1}{N_{t}} \mathbf{h h}^{\dagger} \stackrel{\text { a.s. }}{\rightarrow} \Omega$, as $N_{t} \rightarrow \infty$. In essence, in the large-antenna regime the channel behaves equivalently to an AWGN channel with SNR $\Omega \rho$.

In Fig. 1, the simulated effective rate $\mathcal{R}(\rho, \theta)$ is plotted against the average transmit SNR, $\rho$. The outputs of a MonteCarlo simulator are compared with the exact analytical expression of Proposition 1 and the upper bounds of (10) and (11), respectively. The match between theory and simulation is excellent in all cases under consideration. More importantly, the effective rate is systematically reduced as the QoS requirements become more stringent, i.e., $A$ gets larger. This is 
consistent with the results reported in [8], [10], [11]. Further, a smaller value of $A$ makes both bounds tighter. For example, for $A=0.5$, both bounds become almost exact across the entire SNR range. This implies that they can very efficiently approximate the effective rate for loose delay constraints.

The above results, though exact, provide limited physical insights into the implications of the system parameters on the effective rate. On this basis, we hereafter consider the asymptotically low and high-SNR regimes. We begin with the former regime:

2) Low-SNR analysis: Following the generic methodology of [10], we can assess the low-SNR performance via a secondorder expansion of the effective rate around $\rho \rightarrow 0^{+}$according to

$$
\mathcal{R}(\rho, \theta)=\dot{\mathcal{R}}(0, \theta) \rho+\ddot{\mathcal{R}}(0, \theta) \frac{\rho^{2}}{2}+o\left(\rho^{2}\right)
$$

where $\dot{\mathcal{R}}(\rho, \theta)$ and $\ddot{\mathcal{R}}(\rho, \theta)$ denote the first and second order derivatives of the effective rate (4) with respect to the SNR $\rho$. We point out that these derivative expressions are inherently related with the notions of the minimum normalized energy per information bit to reliably convey any positive rate and the wideband slope respectively, originally proposed in [25]. For the case of QoS constraints, the latter two metrics are respectively defined as,

$\frac{E_{b}}{N_{0} \text { min }} \triangleq \lim _{\rho \rightarrow 0} \frac{\rho}{\mathcal{R}(\rho, \theta)}=\frac{1}{\dot{\mathcal{R}}(0, \theta)}, \mathcal{S}_{0} \triangleq-\frac{2 \ln 2[\dot{\mathcal{R}}(0, \theta)]^{2}}{\ddot{\mathcal{R}}(0, \theta)}$.

Proposition 2: For Nakagami- $m$ fading, the minimum $\frac{E_{b}}{N_{0}}$ and wideband slope $\mathcal{S}_{0}$ are respectively given by

$$
\begin{aligned}
{\frac{E_{b}}{N_{0} \text { min }}}=\frac{\ln 2}{\Omega} \\
\mathcal{S}_{0}=\frac{2 m N_{t}}{A+1+m N_{t}} .
\end{aligned}
$$

Proof: Omitting explicit details and following a similar line of reasoning as in [11, Appendix I], the first and secondorder derivatives in (19) are given by

$$
\begin{aligned}
& \dot{\mathcal{R}}(0, \theta)=\frac{1}{N_{t} \ln 2} \mathrm{E}\left\{\mathbf{h h}^{\dagger}\right\} \\
& \ddot{\mathcal{R}}(0, \theta)=-\frac{A+1}{N_{t}^{2} \ln 2} \mathrm{E}\left\{\left(\mathbf{h h}^{\dagger}\right)^{2}\right\}+\frac{A}{N_{t}^{2} \ln 2}\left(\mathrm{E}\left\{\mathbf{h} \mathbf{h}^{\dagger}\right\}\right)^{2} .
\end{aligned}
$$

We can then use the following results on the traces of $N_{r} \times N_{t}$ MIMO Nakagami- $m$ fading matrices [26]

$$
\begin{aligned}
\mathrm{E}\left\{\operatorname{tr}\left(\mathbf{H H}^{\dagger}\right)\right\} & =N_{r} N_{t} \Omega \\
\mathrm{E}\left\{\operatorname{tr}\left(\mathbf{H H}^{\dagger}\right)^{2}\right\} & =N_{r} N_{t} \Omega^{2}\left(N_{t}+N_{r}-1+1 / m\right) .
\end{aligned}
$$

Combining (22), (23) with (24)-(25) for $N_{r}=1$, we can obtain the desired result after some basic algebraic manipulations.

Interestingly, $\frac{E_{b}}{N_{0} \min }$ is independent of the $m$-factor and delay constraints, whereas $\mathcal{S}_{0}$ is independent of the average power $\Omega$. We note that $\mathcal{S}_{0}$ is an increasing function in $N_{t}$ and $m$ and as such it obtains its minimum value for $m=0.5$ and

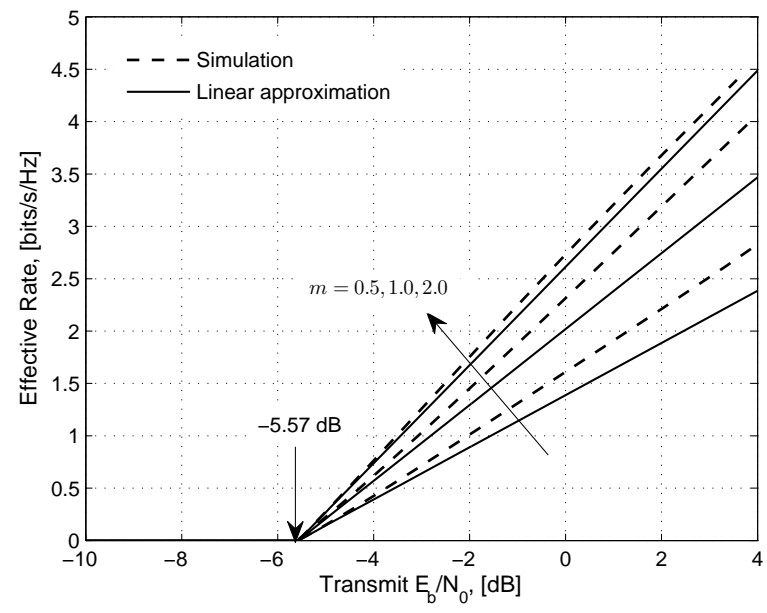

Fig. 2. Low-SNR effective rate and analytical linear approximation against the transmit energy per bit for Nakagami- $m$ fading $\left(N_{t}=6, A=4, \Omega=\right.$ $2.5)$.

its maximum value for $m \rightarrow \infty$. Thus, we have that

$$
\frac{N_{t}}{A+1+\frac{N_{t}}{2}} \leq \mathcal{S}_{0} \leq 2 .
$$

On the other hand, $\mathcal{S}_{0}$ is a monotonically decreasing function in $A$, which implies that delay constraints reduce wideband slope and, in turn, the effective rate.

In Fig. 2, the simulated low-SNR effective rate and the analytical linear approximation (18) are depicted against the transmit energy per bit $E_{b} / N_{0}$, for $N_{t}=6, A=4$, and $\Omega=2.5$. The graph validates the accuracy of our analytical expressions in (20)-(21). As anticipated, the fading parameter $m$ affects the rate performance through the wideband slope but not through the minimum $\frac{E_{b}}{N_{0}}$. In addition, higher $m$ values yield higher rate, although the gap between the corresponding curves decreases as $m$ increases which implies that its effect becomes less pronounced.

3) High-SNR analysis: In the high-SNR regime, we can invoke the following affine expansion of the effective rate, which was originally applied in the context of multiple access systems with random spreading [27] and thereafter in the analysis of MIMO systems [28]:

$$
\mathcal{R}(\rho, \theta)=\mathcal{S}_{\infty}\left(\log _{2} \rho-\mathcal{L}_{\infty}\right)+o(1)
$$

where $\mathcal{S}_{\infty}$ is the so-called high-SNR slope in bits/s/Hz per $3-\mathrm{dB}$ units, given by

$$
\mathcal{S}_{\infty}=\lim _{\rho \rightarrow \infty} \frac{\mathcal{R}(\rho, \theta)}{\log _{2} \rho}
$$

while $\mathcal{L}_{\infty}$ is the zero-th order term or high-SNR power offset, in $3-\mathrm{dB}$ units, given by

$$
\mathcal{L}_{\infty}=\lim _{\rho \rightarrow \infty}\left(\log _{2} \rho-\frac{\mathcal{R}(\rho, \theta)}{\mathcal{S}_{\infty}}\right) .
$$

Proposition 3: For Nakagami- $m$ fading, the high-SNR slope $\mathcal{S}_{\infty}$ is given as

$$
\mathcal{S}_{\infty}= \begin{cases}1, & A \leq m N_{t} \\ \frac{m N_{t}}{A}, & A>m N_{t}\end{cases}
$$




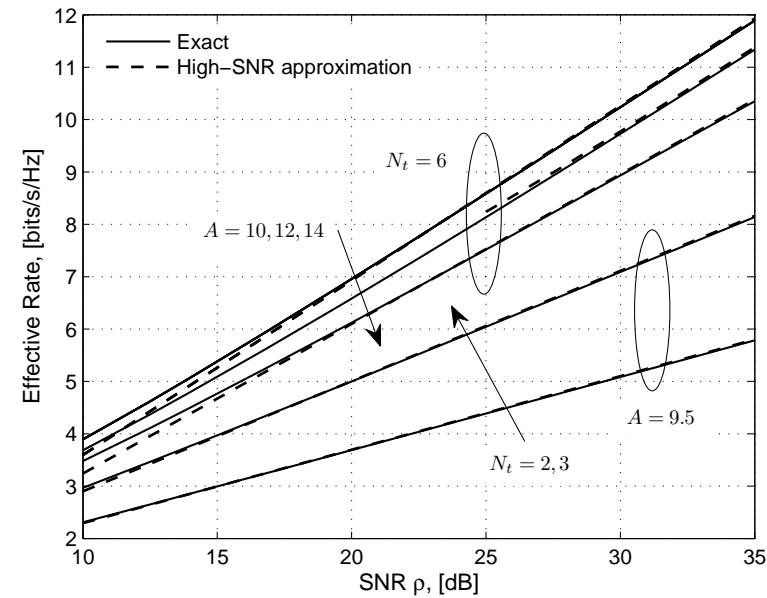

Fig. 3. Exact analytical effective rate and high-SNR approximation against the SNR for Nakagami- $m$ fading $(m=2, \Omega=2.5)$.

while the power offset $\mathcal{L}_{\infty}$ is given by (31) at the bottom of the page, where $\psi(x)$ is the digamma function [17, Eq. (8.360.1)] and $\gamma=0.577216$ is the Euler constant.

Proof: A detailed proof is given in Appendix A.

The above results indicate that the high-SNR slope is independent of the average power $\Omega$, whereas a higher $\Omega$ tends to increase the effective rate by reducing the power offset. It is interesting to note the similarity of the presented expressions with those reported for Rayleigh fading channels in [11]. In Fig. 3, the exact analytical effective rate expression (7) is plotted against the high-SNR approximation of Proposition 3.

Clearly, the high-SNR approximations are sufficiently tight and become exact even at moderate SNR values. This implies that they can efficiently predict the effective rate over a wide SNR range. When $A<m N_{t}$, we can not increase the highSNR slope by increasing $N_{t}$. Yet, a larger $N_{t}$ will effectively reduce the power offset, thereby yielding higher effective rate. Similarly, we can clearly see that when $A$ increases above $m N_{t}$ it has a noticeable impact on the effective rate due to the smaller high-SNR slope. For large and fixed $A$ such that $A>m N_{t}$, an increase in the number of transmit antennas $N_{t}$ can compensate for the loss due to the delay constraints. For example, adding $n$ transmit antennas will linearly increase the high-SNR slope by $n m / A$.

Remark 1: Our numerical results demonstrated that for $A \gg m N_{t}$, the simulated effective rate may diverge for very large values of the SNR (e.g., $\rho>40 \mathrm{~dB}$ ). To address this problem, one can use the following alternative expression for the asymptotic behavior of the Tricomi function [18, Eq.
$(13.5 .10)]$

$$
U(a ; b ; z)=\frac{\Gamma(1-b)}{\Gamma(a)}+\mathcal{O}\left(|z|^{1-b}\right), 0<b<1 .
$$

Applying (32) on Proposition 3, we can obtain the same results for (30) and (31) with the only difference pertaining to their last branch, which instead of $m N_{t}<A$ will be defined as $m N_{t}<A<m N_{t}+1$. This additional constraint on the values of $A$ guarantees that the numerical results remain consistent.

\section{B. Rician fading channels}

The Rician fading model is suitable when the radio channel is dominated by a direct line-of-sight or specular component. This scenario occurs frequently in microcellular urban and suburban land-mobile [29], as well as picocellular indoor environments [30]. Under these circumstances, the entries of the channel vector $\mathbf{h}$ are assumed to be i.i.d. Rician RVs with parameters $K$ and $\Omega$, where $K$ represents the Rician $K$-factor and $\Omega$ the average fading power. The p.d.f. of $x=\left|h_{k}\right|^{2}$ $\left(k=1, \ldots, N_{t}\right)$ is given by [12, Eq. (2.16)]

$$
\begin{aligned}
p(x) & =\frac{(1+K) e^{-K}}{\Omega} \exp \left(-\frac{(K+1) x}{\Omega}\right) \\
& \times I_{0}\left(2 \sqrt{\frac{K(K+1) x}{\Omega}}\right), \quad K, \Omega \geq 0
\end{aligned}
$$

where $I_{\nu}(x)$ is the $\nu$-th order modified Bessel function of the first kind [17, Eq. (8.405.1)].

1) Exact analysis: We first obtain the exact $\mathcal{R}(\rho, \theta)$ as follows:

Proposition 4: For Rician fading, the effective rate of MISO channels is given by

$$
\begin{aligned}
\mathcal{R}(\rho, \theta) & =\log _{2}\left(\frac{\Omega \rho}{(K+1) N_{t}}\right)+\frac{K N_{t}}{A \ln 2} \\
& -\frac{1}{A} \log _{2}\left(\sum_{n=0}^{\infty} \frac{\left(K N_{t}\right)^{n}}{\Gamma(n+1)}\right. \\
& \left.\times U\left(A ; A+1-N_{t}-n ; \frac{(K+1) N_{t}}{\Omega \rho}\right)\right) .
\end{aligned}
$$

Proof: The proof relies on the properties of non-central chi-square distributions. For the particular case under consideration, we can directly use the following expression [31, Eq. (5)] for the p.d.f. of the sum of $N_{t}$ squared i.i.d. Rician RVs, $z=\sum_{k=1}^{N_{t}}\left|h_{k}\right|^{2}$, given by

$$
\mathcal{L}_{\infty}= \begin{cases}\log _{2}\left(\frac{m N_{t}}{\Omega}\right)+\frac{1}{A} \log _{2}\left(\frac{\Gamma\left(m N_{t}-A\right)}{\Gamma\left(m N_{t}\right)}\right), & A<m N_{t} \\ \log _{2}\left(\frac{m N_{t}}{\Omega}\right)+\frac{1}{A} \log _{2}\left(\frac{\ln \left(\frac{\Omega \rho}{m N_{t}}\right)-\psi\left(m N_{t}\right)-2 \gamma}{\Gamma\left(m N_{t}\right)}\right), & A=m N_{t} \\ \log _{2}\left(\frac{m N_{t}}{\Omega}\right)+\frac{1}{m N_{t}} \log _{2}\left(\frac{\Gamma\left(A-m N_{t}\right)}{\Gamma(A)}\right), & A>m N_{t} .\end{cases}
$$




$$
\begin{aligned}
& p(z)=\frac{(K+1) e^{-K N_{t}}}{\Omega}\left(\frac{(K+1) z}{K N_{t} \Omega}\right)^{\frac{N_{t}-1}{2}} \\
& \times \exp \left(-\frac{(K+1) z}{\Omega}\right) I_{N_{t}-1}\left(2 \sqrt{\frac{K(K+1) N_{t} z}{\Omega}}\right) .
\end{aligned}
$$

Substituting (35) into (4) and thereafter using the infinite series representation of $I_{0}(\cdot)$ from [17, Eq. (8.445.1)], we can obtain the desired result after invoking (9).

In order to evaluate (34) we need to truncate the infinite series. We therefore seek to obtain the truncation error which also demonstrates the series' convergence. Assuming that $T_{0}-$ 1 terms are used, the associated truncation error $E_{0}$ can be expressed as

$$
\begin{aligned}
E_{0} & =\sum_{n=T_{0}}^{\infty} \frac{\left(K N_{t}\right)^{n}}{\Gamma(n+1)} U\left(A ; A+1-N_{t}-n ; \frac{(K+1) N_{t}}{\Omega \rho}\right) \\
& <U\left(A ; A+1-N_{t}-T_{0} ; \frac{(K+1) N_{t}}{\Omega \rho}\right) \sum_{n=T_{0}}^{\infty} \frac{\left(K N_{t}\right)^{n}}{\Gamma(n+1)} \\
& =U\left(A ; A+1-N_{t}-T_{0} ; \frac{(K+1) N_{t}}{\Omega \rho}\right) \exp \left(K N_{t}\right) \\
& \times\left(1-\frac{\Gamma\left(T_{0}, K N_{t}\right)}{\Gamma\left(T_{0}\right)}\right)
\end{aligned}
$$

with $\Gamma(p, x)=\int_{x}^{\infty} t^{p-1} e^{-t} d t$ being the upper incomplete gamma function [17, Eq. (8.350.2)]. Note that from (36) to (37) we have used the fact that $U(a, b-n, z)$ is a monotonically decreasing function in $n$, while (38) is a result of $[18$, Eq. (6.5.4)] and [18, Eq. (6.5.29)]

$$
\Gamma(p, x)=\Gamma(p)-x^{p} \Gamma(p) \exp (-x) \sum_{n=0}^{\infty} \frac{x^{n}}{\Gamma(p+n+1)} .
$$

2) Low-SNR analysis: We now investigate the energy efficiency in the low-SNR regime and present tractable results on the parameters dictating the low-SNR performance of MISO Rician fading channels.

Proposition 5: For Rician fading, the minimum $\frac{E_{b}}{N_{0}}$ and wideband slope $\mathcal{S}_{0}$ are respectively given by

$$
\begin{aligned}
\frac{E_{b}}{N_{0} \text { min }} & =\frac{\ln 2}{\Omega} \\
\mathcal{S}_{0} & =\frac{2 N_{t}(K+1)^{2}}{N_{t}(K+1)^{2}+(A+1)(2 K+1)} .
\end{aligned}
$$

Proof: Recalling that $\mathrm{E}\left\{\left|h_{k}\right|^{2}\right\}=\Omega, \forall k=1, \ldots, N_{t}$, we can easily infer that $\mathrm{E}\left\{\mathbf{h h}^{\dagger}\right\}=N_{t} \Omega$. The fourth moment of $\left|h_{k}\right|$ can now be computed according to

$$
\begin{aligned}
& \mathrm{E}\left\{\left|h_{k}\right|^{4}\right\}=\frac{(1+K) e^{-K}}{\Omega} \\
& \times \int_{0}^{\infty} x^{2} \exp \left(-\frac{(K+1) x}{\Omega}\right) I_{0}\left(2 \sqrt{\frac{K(K+1) x}{\Omega}}\right) d x \\
& =\frac{\left(2+4 K+K^{2}\right) \Omega^{2}}{(K+1)^{2}}
\end{aligned}
$$

where we have used [13, Eq. (50)] to evaluate the integral. As

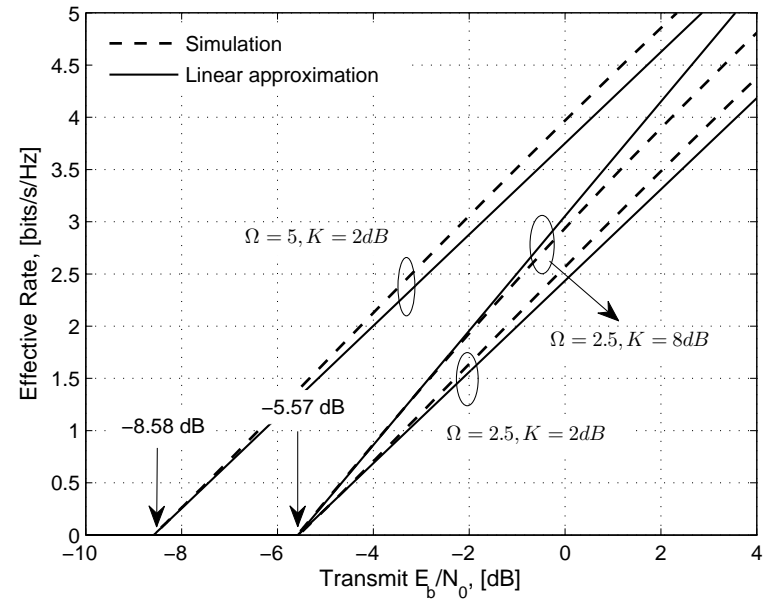

Fig. 4. Low-SNR effective rate and analytical linear approximation against the transmit energy per bit for Rician fading $\left(N_{t}=6, A=4\right)$.

a next step, (42) can be used in the following way

$$
\begin{aligned}
& \mathrm{E}\left\{\left(\mathbf{h h}^{\dagger}\right)^{2}\right\}=\mathrm{E}\left\{\left(\sum_{k=1}^{N_{t}}\left|h_{k}\right|^{2}\right)^{2}\right\} \\
& =\sum_{k=1}^{N_{t}} \mathrm{E}\left\{\left|h_{k}\right|^{4}\right\}+\sum_{k=1}^{N_{t}} \sum_{j=1, j \neq k}^{N_{t}} \mathrm{E}\left\{\left|h_{k}\right|^{2}\left|h_{j}\right|^{2}\right\} \\
& =\frac{N_{t} \Omega^{2}}{(K+1)^{2}}\left(2+4 K+K^{2}\right)+N_{t}\left(N_{t}-1\right) \Omega^{2} \\
& =N_{t} \Omega^{2}\left(N_{t}+\frac{1}{K+1}+\frac{K}{(K+1)^{2}}\right) .
\end{aligned}
$$

From (43) to (44) we have exploited the independence of $\left|h_{k}\right|^{2}$ and $\left|h_{j}\right|^{2}$. The proof then follows trivially by invoking (22)(23) and simplifying.

Similar to the Nakagami- $m$ case, $\frac{E_{b}}{N_{0}}$ min is independent of the $K$-factor and delay constraints, while the wideband slope is an increasing function in $K$, satisfying

$$
\frac{2 N_{t}}{A+1+N_{t}} \leq \mathcal{S}_{0} \leq 2
$$

The lower bound in (46) is attained for $K=0$ (i.e. Rayleigh fading), while the upper bound is approached for $K \rightarrow \infty$ (i.e. AWGN channel). It is noteworthy that for the case of no delay constraints $(A=0)$, the wideband slope reduces to

$$
\mathcal{S}_{0}=\frac{2 N_{t}(K+1)^{2}}{\left(N_{t}+1\right)(2 K+1)+N_{t} K^{2}}
$$

which coincides with [32, Eq. (18)]. It is also worth mentioning that $S_{0}$ is a monotonically decreasing function in $A$, since we have that

$$
\frac{d \mathcal{S}_{0}}{d A}=-\frac{2 N_{t}(2 K+1)(K+1)^{2}}{\left((2 K+1)(A+1)+N_{t}(K+1)^{2}\right)^{2}}<0 .
$$

This validates that strict delay constraints tend to reduce the effective rate.

Figure 4 more closely investigates the low-SNR performance of Rician fading channels. As for the Nakagami- $m$ case, the linear approximations remain sufficiently tight across a wide range of SNR values. It is readily seen that a $50 \%$ 
increase in the average fading power $\Omega$ reduces the minimum energy per bit by $3 \mathrm{~dB}$. Meanwhile, a higher $K$-factor leaves $\frac{E_{b}}{N_{0}}$ unaffected but still increases the effective rate, through an enhanced $\mathcal{S}_{0}$. This increase is more pronounced for smaller values of $K$. For example, an increase in $K$ from 0 to 1 will increase the wideband slope by $1+(A+1) /\left(4 N_{t}+3(A+1)\right)$. We note that these results are in line with those reported in [32].

3) High-SNR analysis: The presence of a Tricomi function in the effective rate expression (34) does not allow straightforward algebraic manipulations. As such, the useful parametrization in terms of high-SNR slope and power offset can hardly be implemented. Yet, by considering the initial expression (4) and keeping only the dominant term therein as $\rho \rightarrow \infty$, we can obtain the following tractable result.

Proposition 6: For Rician fading, the effective rate of MISO channels at high SNRs and for $A<N_{t}$ is approximated by

$$
\begin{aligned}
& \mathcal{R}^{\infty}(\rho, \theta) \approx \log _{2}\left(\frac{\rho \Omega}{(K+1) N_{t}}\right)+\frac{K N_{t}}{A \ln 2} \\
& -\frac{1}{A} \log _{2}\left(\frac{\Gamma\left(N_{t}-A\right)}{\Gamma\left(N_{t}\right)}{ }_{1} F_{1}\left(N_{t}-A ; N_{t} ; K N_{t}\right)\right) .
\end{aligned}
$$

Proof: By taking $\rho$ large in (4), the proof boils down to the computation of the $A$-th negative moment of $z$, $\mathrm{E}\left\{z^{-A}\right\}$. As a next step, we express the Bessel function in (35) via a hypergeometric function according to [21, Eq. (03.02.26.0002.01)]

$$
I_{\nu}(x)=\frac{1}{\Gamma(\nu+1)}\left(\frac{x}{2}\right)^{\nu}{ }_{0} F_{1}\left(-; \nu+1 ; \frac{x^{2}}{4}\right) .
$$

Combining (50) with (35), we can obtain the desired result by invoking the following integral identity [17, Eq. (7.522.5)]

$$
\begin{array}{r}
\int_{0}^{\infty} e^{-x} x^{\nu-1}{ }_{p} F_{q}\left(a_{1}, \ldots, a_{p} ; b_{1}, \ldots, b_{q} ; \alpha x\right) \\
\quad=\Gamma(\nu)_{p+1} F_{q}\left(\nu, a_{1}, \ldots, a_{p} ; b_{1}, \ldots, b_{q} ; \alpha\right)
\end{array}
$$

for $p<q$ and $\operatorname{Re}(\nu)>0$, and simplifying. Note that the condition on the arguments of (51) is satisfied in our setting by taking $A<N_{t}$.

The above result indicates that the high-SNR slope is 1 , thereby reflecting the same observations made for the Nakagami- $m$ case. From Proposition 6 , it can be also shown that the high-SNR effective rate is a monotonically increasing function in the Rician $K$-factor. This is anticipated, since larger values of $K$ reduce the signal's envelope fluctuations, making fading manifestations more deterministic. We finally note that the effective rate grows logarithmically with the SNR, when $\rho \rightarrow \infty$.

\section{Generalized- $\mathcal{K}$ fading channels}

The last fading model we are considering is the so-called generalized- $\mathcal{K}$ fading model. This is a generic composite model that encompasses both small-scale fading (modeled via the Nakagami- $m$ distribution) and large-scale fading (modeled via the gamma distribution). Its main characteristic is that is analytically friendlier than the classical Nakagami$\mathrm{m} /$ lognormal model, thereby lending itself into tractable manipulations. In parallel, it has been demonstrated to effectively approximate most of the fading and shadowing phenomena occurring in wireless channels [33]-[36]. In this case, the entries of the channel vector $\mathbf{h}$ are assumed to be i.i.d. generalized- $\mathcal{K}$ $\mathrm{RVs}^{2}$ with parameters $m, \kappa$ and $\Omega$, where $\kappa \geq 0, m \geq 0.5$ are the distribution shaping parameters and $\Omega$ is the average fading power. Then, the p.d.f. of $x=\left|h_{k}\right|^{2}\left(k=1, \ldots, N_{t}\right)$ is given by [34, Eq. (2)]

$$
\begin{aligned}
p(x) & =\frac{2 x^{\frac{\kappa+m}{2}-1}}{\Gamma(m) \Gamma(\kappa)}\left(\frac{\kappa m}{\Omega}\right)^{\frac{\kappa+m}{2}} \\
& \times K_{\kappa-m}\left(2 \sqrt{\frac{\kappa m}{\Omega} x}\right), \quad \kappa, \Omega \geq 0, m \geq 0.5
\end{aligned}
$$

where $K_{\nu}(x)$ denotes the $\nu$-th order modified Bessel function of the second kind [17, Eq. (8.407.1)].

1) Exact analysis: In general, an exact analysis for the case of generalized- $\mathcal{K}$ fading is cumbersome since, up to date, the exact distribution of the sum of generalized- $\mathcal{K} \mathrm{RVs}$ is not known in closed-form. However, we can use the tight approximation proposed in [38] and obtain analytical expressions for the most important figures of merit. ${ }^{3}$

Proposition 7: For generalized- $\mathcal{K}$ fading, the effective rate of MISO channels is approximately given by

$$
\mathcal{R}(\rho, \theta) \approx-\frac{1}{A} \log _{2}\left({ }_{3} F_{0}\left(A, \hat{\kappa}, \hat{m} ;-;-\frac{\hat{\Omega} \rho}{\hat{\kappa} \hat{m} N_{t}}\right)\right)
$$

where $\hat{m}, \hat{\kappa}$ and $\hat{\Omega}$ are defined as

$$
\begin{aligned}
& \hat{m}=m N_{t} \\
& \hat{\kappa}=\kappa N_{t}+\left(N_{t}-1\right) \frac{-0.127-0.95 \kappa-0.0058 m}{1+0.00124 \kappa+0.98 m} \\
& \hat{\Omega}=\Omega N_{t} .
\end{aligned}
$$

Proof: Using [38], the p.d.f. of $z=\sum_{k=1}^{N_{t}}\left|h_{k}\right|^{2}$ can be approximated as

$$
p(z) \approx \frac{2 z^{\frac{\hat{\kappa}+\hat{m}}{2}-1}}{\Gamma(\hat{m}) \Gamma(\hat{\kappa})}\left(\frac{\hat{\kappa} \hat{m}}{\hat{\Omega}}\right)^{\frac{\hat{\kappa}+\hat{m}}{2}} K_{\hat{\kappa}-\hat{m}}\left(2 \sqrt{\frac{\hat{\kappa} \hat{m}}{\hat{\Omega}} z}\right) .
$$

Substituting (57) into (4), we end up with the following approximating integral expression for the effective rate

$$
\begin{aligned}
& \mathcal{R}(\rho, \theta) \approx-\frac{1}{A} \log _{2}\left(\frac{2}{\Gamma(\hat{m}) \Gamma(\hat{\kappa})}\left(\frac{\hat{\kappa} \hat{m}}{\hat{\Omega}}\right)^{\frac{\hat{\kappa}+\hat{m}}{2}}\right. \\
& \left.\times \int_{0}^{\infty}\left(1+\frac{\rho}{N_{t}} z\right)^{-A} z^{\frac{\hat{\kappa}+\hat{m}}{2}-1} K_{\hat{\kappa}-\hat{m}}\left(2 \sqrt{\frac{\hat{\kappa} \hat{m}}{\hat{\Omega}} z}\right) d z\right) .
\end{aligned}
$$

To evaluate the integral in (58), we first express the integrands $\left(1+\frac{\rho}{N_{t}} z\right)^{-A}$ and $K_{\hat{\kappa}-\hat{m}}\left(2 \sqrt{\frac{\hat{\kappa} \hat{m}}{\hat{\Omega}} z}\right)$ in terms of Meijer's- $G$ functions with the help of [24, Eq. (8.4.2.5)] and

\footnotetext{
${ }^{2}$ The assumption of i.i.d. large-scale fading is sufficiently realistic for distributed antenna systems or when the antenna separation is large [12]. Nevertheless, it was recently shown, via real measurement data [37], that even for arrays with colocated antennas, shadowing can be independent across the array.

${ }^{3} \mathrm{~A}$ detailed discussion about the tightness of this and previous approximations can be found in [38].
} 
[24, Eq. (8.4.23.1)], respectively

$$
\begin{aligned}
&\left(1+\frac{\rho}{N_{t}} z\right)^{-A}=\frac{1}{\Gamma(A)} G_{1,1}^{1,1}\left[\frac{\rho}{N_{t}} z \mid \begin{array}{c}
1-A \\
0
\end{array}\right] \\
& K_{\hat{\kappa}-\hat{m}}\left(2 \sqrt{\left.\frac{\hat{\kappa} \hat{m}}{\hat{\Omega}} z\right)}=\frac{1}{2} G_{0,2}^{2,0}\left[\left.\frac{\hat{\kappa} \hat{m}}{\hat{\Omega}} z\right|_{\frac{\hat{\kappa}-\hat{m}}{2},-\frac{\hat{\kappa}-\hat{m}}{2}}\right] .\right.
\end{aligned}
$$

Then, combining (59), (60) with (58), and using the identity [24, Eq. (2.24.1.1)], we obtain

$$
\begin{aligned}
\mathcal{R}(\rho, \theta) & \approx-\frac{1}{A} \log _{2}\left(\frac{1}{\Gamma(\hat{m}) \Gamma(\hat{\kappa}) \Gamma(A)}\right. \\
& \left.\times G_{3,1}^{1,3}\left[\frac{\hat{\Omega} \rho}{\hat{\kappa} \hat{m} N_{t}} \mid \begin{array}{c}
1-A, 1-\hat{\kappa}, 1-\hat{m} \\
0
\end{array}\right]\right) .
\end{aligned}
$$

Finally, by applying [24, Eq. (8.4.51.1)] on (61), we arrive at the desired result in (53).

2) Low-SNR analysis: In the low-SNR regime, one can exploit the independence of the channel entries to obtain exact expressions for the minimum $\frac{E_{b}}{N_{0}}$ and wideband slope $\mathcal{S}_{0}$.

Proposition 8: For generalized- $\mathcal{K}$ fading, the minimum $\frac{E_{b}}{N_{0}}$ and wideband slope $\mathcal{S}_{0}$ are respectively given by

$$
\begin{aligned}
{\frac{E_{b}}{N_{0} \text { min }}} & =\frac{\ln 2}{\Omega} \\
\mathcal{S}_{0} & =\frac{2 \kappa m N_{t}}{\kappa m N_{t}+(A+1)(\kappa+m+1)} .
\end{aligned}
$$

Proof: For the evaluation of the expectations in (22) and (23), we use the standard expression for the $n$-th order moment of a generalized- $\mathcal{K} \mathrm{RV}$ with parameters $m, \kappa, \Omega$, given by [38, Eq. (4)]:

$$
\mathrm{E}\left\{\left(\left|h_{k}\right|^{2}\right)^{n}\right\}=\left(\frac{\Omega}{\kappa m}\right)^{n} \frac{\Gamma(\kappa+n) \Gamma(m+n)}{\Gamma(\kappa) \Gamma(m)} .
$$

With this relationship in our hands, we can easily deduce

$$
\begin{aligned}
\mathrm{E}\left\{\mathbf{h h}^{\dagger}\right\} & =N_{t} \Omega \\
\mathrm{E}\left\{\left(\mathbf{h h}^{\dagger}\right)^{2}\right\} & \stackrel{(43)}{=} \frac{N_{t} \Omega^{2}}{\kappa m}(\kappa+1)(m+1)+N_{t}\left(N_{t}-1\right) \Omega^{2} \\
& =\frac{N_{t} \Omega^{2}}{\kappa m}\left(\kappa+m+1+\kappa m N_{t}\right)
\end{aligned}
$$

Combining (22), (23) with (65) and (66), we can obtain the desired result after some basic algebra.

Clearly, the minimum $\frac{E_{b}}{N_{0}}$ is identical with that for the Nakagami- $m$ and Rician fading scenarios. At the same time, it is independent of the shaping parameters $m$ and $\kappa$. As for the cases of Nakagami- $m$ and Rician fading, we can infer that $\mathcal{S}_{0}$ is a monotonically decreasing function in $A$. In addition, it is monotonically increasing in $\kappa$, since we have that

$$
\frac{d \mathcal{S}_{0}}{d \kappa}=\frac{2 m N_{t}(A+1)(m+1)}{\left(\kappa m N_{t}+(\kappa+m+1)(A+1)\right)^{2}}>0 .
$$

Note that in the limit $\kappa \rightarrow \infty$, (63) reduces to (21). This is expected since for $\kappa \rightarrow \infty$, the generalized- $\mathcal{K}$ distribution approximates the Nakagami- $m$ distribution [33]. For the special case of double Rayleigh fading (i.e. $m=\kappa=1$ ), that is frequently used in cascaded multipath fading channels [39],

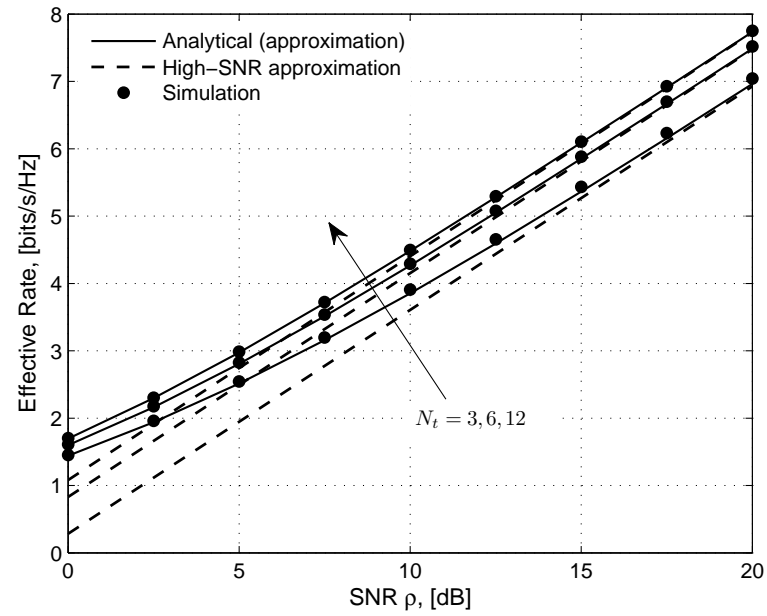

Fig. 5. Simulated effective rate, analytical and high SNR approximations against the SNR for generalized- $\mathcal{K}$ fading $(A=1.5, m=2.0, \kappa=$ $1.45, \Omega=2.5)$.

(63) simplifies to

$$
\mathcal{S}_{0}=\frac{2 N_{t}}{N_{t}+3(A+1)}
$$

3) High-SNR analysis: As for the Rician case, the presence of a hypergeometric function in the effective rate expression (53) renders the high-SNR analysis cumbersome. For this reason, we follow the methodology of Proposition 6 to obtain the following tractable result.

Proposition 9: For generalized- $\mathcal{K}$ fading, the effective rate of MISO channels at high SNRs and for $A<\hat{m}$ and $A<\hat{\kappa}$ is approximated by

$$
\begin{aligned}
\mathcal{R}^{\infty}(\rho, \theta) & \approx \log _{2}\left(\frac{\hat{\Omega} \rho}{\hat{\kappa} \hat{m} N_{t}}\right) \\
& -\frac{1}{A} \log _{2}\left(\frac{\Gamma(\hat{m}-A) \Gamma(\hat{\kappa}-A)}{\Gamma(\hat{m}) \Gamma(\hat{\kappa})}\right) .
\end{aligned}
$$

Proof: By taking $\rho$ large in (4), we have to compute the $A$-th negative moment of $z, \mathrm{E}\left\{z^{-A}\right\}$ as follows

$$
\begin{aligned}
\mathrm{E}\left\{z^{-A}\right\} & =\frac{2}{\Gamma(\hat{m}) \Gamma(\hat{\kappa})}\left(\frac{\hat{\kappa} \hat{m}}{\hat{\Omega}}\right)^{\frac{\hat{\kappa}+\hat{m}}{2}} \\
& \times \int_{0}^{\infty} z^{\frac{\hat{\kappa}+\hat{m}}{2}-A-1} K_{\hat{\kappa}-\hat{m}}\left(2 \sqrt{\frac{\hat{\kappa} \hat{m}}{\hat{\Omega}} z}\right) d z \\
& =\left(\frac{\hat{\kappa} \hat{m}}{\hat{\Omega}}\right)^{A} \frac{\Gamma(\hat{m}-A) \Gamma(\hat{\kappa}-A)}{\Gamma(\hat{m}) \Gamma(\hat{\kappa})}
\end{aligned}
$$

where from (70) to (71) we have used the integration relationship [17, Eq. (6.561.16)] and thereafter simplified. Note that the condition for this integration relationship requires $A<\hat{m}$ and $A<\hat{\kappa}$.

As for the previous fading models, the slope of the effective rate at high SNRs is equal to 1 . We finally note that for the particular case $A=1$, (69) simplifies to

$$
\mathcal{R}^{\infty}(\rho, \theta) \approx \log _{2}\left(\frac{\hat{\Omega} \rho(\hat{m}-1)(\hat{\kappa}-1)}{\hat{\kappa} \hat{m} N_{t}}\right)
$$

which indicates the beneficial impact of $\hat{\kappa}, \hat{m}$, due to the 
reduced fading fluctuations [33], [34].

In Fig. 5, the simulated effective rate is plotted along with the approximating expression for the exact effective rate (53) and the high-SNR approximation in (69). We consider different MISO configurations by increasing the number of transmit antennas $N_{t}$. It is easily seen that the approximation for the exact rate is remarkably tight for all the considered cases and values of SNR, while its tightness is slightly improved for larger $N_{t}$. This validates that the approximation of [38] can accurately model the p.d.f. of the sum of generalized- $\mathcal{K}$ RVs. On similar grounds, the high-SNR approximation becomes exact around $15 \mathrm{~dB}$. We also observe that an increase in $N_{t}$ tends to increase the effective rate, albeit the relative difference between the curves gets steadily smaller.

\section{CONCLUSION}

A plethora of emerging applications, such as VoIP and mobile computing, impose stringent delay constraints that have to be appropriately accounted for, using a suitable metric. Unfortunately, the classical notion of Shannon's ergodic capacity fails to do so. Hence, the concept of effective rate arises which can efficiently characterize communication systems in terms of data rate, delay and delay-violation probability. Yet, most studies reported in this context consider the tractable case of Rayleigh fading channels.

In this paper, a detailed effective rate analysis of MISO systems was presented. In particular, we considered three popular fading models, namely Nakagami- $m$, Rician and generalized- $\mathcal{K}$, which have been exhaustively used in the performance analysis of wireless communication systems. For the considered models, new analytical expressions for the exact effective rate were derived that extend and complement previous results on Rayleigh fading channels. Moreover, we elaborated on the asymptotically low and high-SNR regimes for which simple, closed-form expressions were deduced. By doing so, we were able to obtain additional physical insights into the implications of several parameters (e.g. fading parameters, number of antennas, delay constraints) on the system performance. As a final remark, we highlight the fact that the presented analysis can be extended to other fading models of interest, e.g. Weibull, $\eta-\mu$, and $\kappa-\mu$ [40].

\section{APPENDIX A}

\section{PROOF OF PROPOSITION 3}

The proof relies on the asymptotic properties of Tricomi hypergeometric functions. In particular, with the aid of [18, Eq. (13.5.6)-(13.5.12)], the expressions given in (73) hold for the asymptotic behavior of $U(\cdot)$ in (6).

In order to derive the high-SNR slope, we start from (29) and follow the methodology of [10], yielding (74)-(77) at the bottom of the page. Combining (73) with (77), we can obtain the result in (30) after some simplifications and using the fundamental properties of limits. Likewise, the expressions for the high-SNR power offset $\mathcal{L}_{\infty}$ in (31) are obtained via the definition in (29) and (73). This concludes the proof.

\section{REFERENCES}

[1] D. Gesbert, M. Kountouris, R. W. Heath, Jr., C.-B. Chae, and T. Salzer, "Shifting the MIMO paradigm: From single user to multiuser communications," IEEE Signal Process. Mag., vol. 24, no. 5, pp. 3646, Oct. 2007.

[2] D. Wu and R. Negi, "Effective capacity: A wireless link model for support of quality of service," IEEE Trans. Wireless Commun., vol. 2, no. 4, pp. 630-643, July 2003.

[3] J. Tang and X. Zhang, "Quality-of-service driven power and rate adaptation over wireless links," IEEE Trans. Wireless Commun., vol. 6, no. 8, pp. 3058-3068, Aug. 2007.

[4] —, "Quality-of-service driven power and rate adaptation for multichannel communications over wireless links," IEEE Trans. Wireless Commun., vol. 6, no. 12, pp. 4349-4360, Dec. 2007.

[5] L. Liu, P. Parag, J. Tang, W.-Y. Chen, and J.-F. Chamberland, "Resource allocation and quality of service evaluation for wireless communication systems using fluid models," IEEE Trans. Inf. Theory, vol. 53, no. 5, pp. 1767-1777, May 2007.

[6] L. Liu, P. Parag, and J.-F. Chamberland, "Quality of service analysis for wireless user-cooperation networks," IEEE Trans. Inf. Theory, vol. 53, no. 10 , pp. 3833-3842, Oct. 2007.

$$
\lim _{\rho \rightarrow \infty} U\left(m N_{t} ; m N_{t}+1-A ; \frac{m N_{t}}{\Omega \rho}\right)= \begin{cases}\frac{\Gamma\left(m N_{t}-A\right)}{\Gamma\left(m N_{t}\right)}\left(\frac{m N_{t}}{\Omega \rho}\right)^{A-m N_{t}}, & A<m N_{t} \\ \frac{\ln \left(\frac{\Omega \rho}{m N_{t}}\right)-\psi\left(m N_{t}\right)-2 \gamma}{\Gamma\left(m N_{t}\right)}, & A=m N_{t} \\ \frac{\Gamma\left(A-m N_{t}\right)}{\Gamma(A)}, & A>m N_{t} .\end{cases}
$$

$$
\begin{aligned}
\mathcal{S}_{\infty} & =\lim _{\rho \rightarrow \infty} \frac{\mathcal{R}(\rho, \theta)}{\log _{2} \rho} \\
& =\lim _{\rho \rightarrow \infty} \frac{\log _{2} \rho}{A \log _{2} \rho}-\lim _{\rho \rightarrow \infty} \frac{\frac{1}{A} \log _{2}\left(\rho \int_{0}^{\infty}\left(1+\frac{\rho}{N_{t}} z\right)^{-A} p(z) d z\right)}{\log _{2} \rho} \\
& \stackrel{(6)}{=} \frac{1}{A}-\lim _{\rho \rightarrow \infty} \frac{\frac{1}{A} \log _{2}\left(\rho^{-m N_{t}+1}\left(\frac{m N_{t}}{\Omega}\right)^{m N_{t}} U\left(m N_{t} ; m N_{t}+1-A ; \frac{m N_{t}}{\Omega \rho}\right)\right)}{\log _{2} \rho} \\
& =\frac{m N_{t}}{A}-\lim _{\rho \rightarrow \infty} \frac{\frac{1}{A} \log _{2}\left(\left(\frac{m N_{t}}{\Omega}\right)^{m N_{t}} U\left(m N_{t} ; m N_{t}+1-A ; \frac{m N_{t}}{\Omega \rho}\right)\right)}{\log _{2} \rho} .
\end{aligned}
$$


[7] M. C. Gursoy, D. Qiao, and S. Velipasalar, "Analysis of energy efficiency in fading channels under QoS constrains," IEEE Trans. Wireless Commun., vol. 8, no. 8, pp. 4252-4263, Aug. 2009.

[8] L. Liu and J.-F. Chamberland, "On the effective capacity of multiantenna Gaussian channels," in Proc. IEEE Int. Sym. Inf. Theory (ISIT), Toronto, ON, July 2008, pp. 2583-2587.

[9] E. A. Jorswieck, R. Mochaourab, and M. Mittelbach, "Effective capacity maximization in multi-antenna channels with covariance feedback," in Proc. IEEE Int. Conf. Commun. (ICC), Dresden, Germany, June 2009.

[10] M. C. Gursoy, "MIMO wireless communications under statistical queueing constrains," IEEE Trans. Inf. Theory, vol. 57, no. 9, pp. 5897-5917, Sept. 2011.

[11] C. Zhong, T. Ratnarajah, K.-K. Wong, and M.-S. Alouini, "Effective capacity of correlated MISO channels," in Proc. IEEE Int. Conf. Commun. (ICC), Kyoto, Japan, June 2011.

[12] M. K. Simon and M.-S. Alouini, Digital Communication over Fading Channels, 2nd Ed., NY, John Wiley \& Sons, 2005.

[13] M. Nakagami, "The $m$-distribution-A general formula of intensity distribution of rapid fading," in Statistical Methods in Radio Wave Propagation, W. C. Hoffman Ed., pp. 3-36, Oxford, U.K.: Pergamon, 1960.

[14] H. Suzuki, "A statistical model for urban radio propagation," IEEE Trans. Commun., vol. 25, no. 7, pp. 673-680, May 1974.

[15] A. U. Sheikh, M. Handforth, and M. Abdi, "Indoor mobile radio channel at 946 MHz: Measurements and modeling," in Proc. IEEE Veh. Technol. Conf. (VTC), Secaucus, NJ, May 1993, pp. 73-76.

[16] M. Matthaiou, D. I. Laurenson, and J. S. Thompson, "A MIMO channel model based on the Nakagami-faded spatial eigenmodes," IEEE Trans. Ant. Propag., vol. 56, no. 5, pp. 1494-1497, May 2008.

[17] I. S. Gradshteyn and I. M. Ryzhik, Table of Integrals, Series, and Products, Seventh Ed. Academic Press, San Diego, 2007.

[18] M. Abramowitz and I. A. Stegun, Handbook of Mathematical Functions with Formulas, Graphs, and Mathematical Tables, Ninth Ed., NY, Dover, 1970.

[19] M. Evans, N. Hastings, and B. Peacock, Statistical Distributions, 3rd ed. NY: Wiley, 2000.

[20] M. Kang and M.-S. Alouini, "Capacity of MIMO Rician channels," IEEE Trans. Wireless Commun., vol. 5, no. 1, pp. 112-122, Jan. 2006.

[21] Wolfram, "The Wolfram functions site," [Online]. Available: http:// functions.wolfram.com, 2012.

[22] H. Shin and J. Lee, "Capacity of multiple-antenna fading channels: Spatial fading correlation, double scattering, and keyhole," IEEE Trans. Inf. Theory, vol. 49, no. 10, pp. 2636-2647, Oct. 2003.

[23] C. Zhong, M. Matthaiou, G. K. Karagiannidis, and T. Ratnarajah, "Generic ergodic capacity bounds for fixed-gain AF dual-hop relaying systems," IEEE Trans. Veh. Technol., vol. 60, no. 8, pp. 3814-3824, Oct. 2011.

[24] A. P. Prudnikov, Y. A. Brychkov, and O. I. Marichev, Integrals and Series, Volume 3: More Special Functions. NY: Gordon and Breach, 1990.

[25] S. Verdú, "Spectral efficiency in the wideband regime," IEEE Trans. Inf. Theory, vol. 48, no. 6, pp. 1319-1343, June 2002.

[26] C. Zhong, S. Jin, T. Ratnarajah, and K.-K. Wong, "On the capacity of non-uniform phase MIMO Nakagami- $m$ fading channels," IEEE Commun. Lett., vol. 14, no. 6, pp. 536-538, June 2010.

[27] S. Shamai (Shitz) and S. Verdú, "The impact of frequency-flat fading on the spectral efficiency of CDMA," IEEE Trans. Inf. Theory, vol. 47, no. 4, pp. 1302-1327, May 2001.

[28] A. Lozano, A. M. Tulino, and S. Verdú, "High-SNR power offset in multiantenna communications," IEEE Trans. Inf. Theory, vol. 51, no. 12, pp. 4134-4151, Dec. 2005.

[29] K. A. Stewart, G. P. Labedz, and K. Sohrabi, "Wideband channel measurements at $900 \mathrm{MHz}$," in Proc. IEEE Veh. Technol. Conf. (VTC), Chicago, July 1995, pp. 236-240.

[30] R. Bultitude, S. A. Mahmoud, and W. A. Sullivan, "A comparison of indoor radio propagation characteristics at $910 \mathrm{MHz}$ and $1.75 \mathrm{GHz}$," IEEE J. Sel. Areas Commun., vol. 7, no. 1, pp. 20-30, Jan. 1989.

[31] J. Hu and N. C. Beaulieu, "Accurate closed-form approximations to Ricean sum distributions and densities," IEEE Commun. Lett., vol. 9, no. 2, pp. 133-135, Feb. 2005.

[32] A. Lozano, A. M. Tulino, and S. Verdú, "Multiple-antenna capacity in the low-power regime," IEEE Trans. Inf. Theory, vol. 49, no. 10, pp. 2527-2544, Oct. 2003.

[33] P. M. Shankar, "Error rates in generalized shadowed fading channels," Wireless Pers. Commun., vol. 28, no. 3, pp. 233-238, Feb. 2004.

[34] P. S. Bithas, N. C. Sagias, P. T. Mathiopoulos, G. K. Karagiannidis, and A. A. Rontogiannis, "On the performance analysis of digital communications over generalized- $K$ fading channels," IEEE Commun Lett., vol. 10, no. 5, pp. 353-355, May 2006.

[35] A. Laourine, M.-S. Alouini, S. Affes, and A. Stéphenne, "On the capacity of generalized- $K$ fading channels," IEEE Trans. Wireless Commun., vol. 7, no. 7, pp. 2441-2445, July 2008.

[36] M. Matthaiou, N. D. Chatzidiamantis, G. K. Karagiannidis, and J. A. Nossek, "On the capacity of generalized- $K$ fading MIMO channels," IEEE Trans. Signal Process., vol. 58, no. 11, pp. 5939-5944, Nov. 2010.

[37] S. Payami and F. Tufvesson, "Channel measurements and analysis for very large array systems at $2.6 \mathrm{GHz}$," in Proc. Europ. Conf. Anten. Propag. (EuCAP), Prague, Czech Republic, Mar. 2012.

[38] N. D. Chatzidiamantis and G. K. Karagiannidis, "On the distribution of the sum of gamma-gamma variates and applications in RF and optical wireless communications," IEEE Trans. Commun., vol. 59, no. 5, pp. 1298-1308, May 2011.

[39] M. Uysal, "Diversity analysis of space-time coding in cascaded Rayleigh fading channels," IEEE Commun. Lett., vol. 10, no. 3, pp. 165-167, Mar. 2006.

[40] D. B. da Costa and M. D. Yacoub, "Accurate approximation to the sum of generalized random variables and applications in the performance analysis of diversity systems," IEEE Trans. Commun., vol. 57, no. 5, pp. 1271-1274, May 2009.

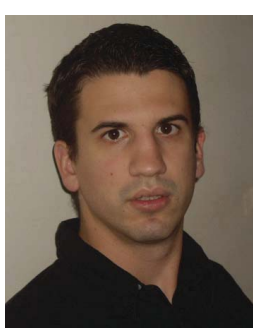

Michail Matthaiou (S'05-M'08) was born in Thessaloniki, Greece in 1981. He obtained the Diploma degree (5 years) in Electrical and Computer Engineering from the Aristotle University of Thessaloniki, Greece in 2004. He then received the M.Sc. (with distinction) in Communication Systems and Signal Processing from the University of Bristol, U.K. and Ph.D. degrees from the University of Edinburgh, U.K. in 2005 and 2008, respectively. From September 2008 through May 2010, he was with the Institute for Circuit Theory and Signal Processing, Munich University of Technology (TUM), Germany working as a Postdoctoral Research Associate. In June 2010, he joined Chalmers University of Technology, Sweden as an Assistant Professor and in 2011 he was awarded the Docent title. His research interests span signal processing for wireless communications, random matrix theory and multivariate statistics for MIMO systems, and performance analysis of fading channels.

Dr. Matthaiou is the recipient of the 2011 IEEE ComSoc Young Researcher Award for the Europe, Middle East and Africa Region and a co-recipient of the 2006 IEEE Communications Chapter Project Prize for the best M.Sc. dissertation in the area of communications. He was an Exemplary Reviewer for IEEE COMMUNICATIONS LETTERS for 2010. He has been a member of Technical Program Committees for several IEEE conferences such as GLOBECOM, DSP, etc. He currently serves as an Associate Editor for the IEEE TRANSACTIONS ON COMMUNICATIONS, IEEE COMMUNICATIONS LETTERS and as a Lead Guest Editor of the special issue on "Large-scale multiple antenna wireless systems" of the IEEE JOURNAL ON SELECTED AREAS In COMMUNICATIONS. He is an associate member of the IEEE Signal Processing Society SPCOM technical committee. 


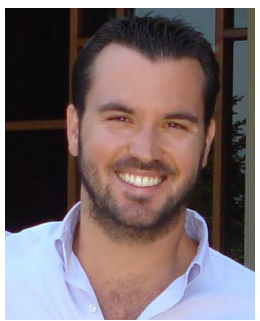

George C. Alexandropoulos (S'07-M'10) was born in Athens, Greece in 1980. He received the Diploma degree (5 years) in Computer Engineering and Informatics, the M.A.Sc. degree (with distinction) in Signal Processing and Communications, and the Ph.D. degree in Wireless Communications from University of Patras (UoP), School of Engineering (SE), Computer Engineering and Informatics Department (CEID), Rio-Patras, Greece in 2003, 2005, and 2010, respectively. From 20012010 he has been a research fellow at the Signal Processing and Communications Laboratory, UoP, SE, CEID, Rio-Patras, Greece. During 2006-2010 he was with the National Centre for Scientific Research-"Demokritos," Athens, Greece, where he was a Ph.D. scholar at the Wireless Communications Laboratory of the Institute of Informatics and Telecommunications. From 2007-2011 he has been affiliated with the National Observatory of Athens, Institute for Space Applications and Remote Sensing, Athens, Greece, where he has participated in several national and European R\&D projects. During the summer semester of 2011 he has been an adjunct lecturer at the University of Peloponnese, Department of Telecommunications Science and Technology, Tripolis, Greece. Currently, he is a researcher at the Athens Information Technology (AIT), Athens, Greece and a member of its Broadband Wireless and Sensor Networks research team.

Dr. G. C. Alexandropoulos' research interests include cooperative and cognitive radio systems, fading channels, MIMO techniques, and signal processing for wireless communications. He currently serves a member of the Editorial Advisory Board of Recent Patents on Telecommunications, Bentham Science Publishers and acts as a reviewer for several international journals and conferences (including IEEE and IET). He has received a postgraduate scholarship from the Operational Programme for Education and Initial Vocational Training II, Ministry of Education, Lifelong Learning, and Religious Affairs, Greek Republic, a student travel grant for the IEEE GLOBECOM 2010 in Miami, Florida, USA, and the best Ph.D. thesis award 2010 by the Informatics and Telematics Institute, Thessaloniki, Greece. He is a member of the IEEE, the IEEE Communications Society and the Technical Chamber of Greece.

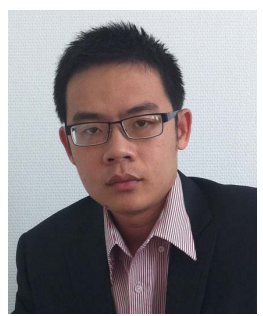

Hien Quoc Ngo received the B.S. degree in Electrical Engineering, major Telecommunications from Ho Chi Minh City University of Technology, Vietnam, in 2007, and the M.S. degree in Electronics and Radio Engineering from Kyung Hee University, Korea, in 2010. From 2008 to 2010, he was with the Communication and Coding Theory Laboratory, Kyung Hee University, where he did research on wireless communication and information theory, in particular cooperative communications, game theory and network connectivity. Since April 2010, he is a Ph.D. student of the Division for Communication Systems in the Department of Electrical Engineering (ISY) at Linköping University (LiU) in Linköping, Sweden. His current research interests include MIMO systems with very large antenna arrays, cooperative communications, and interference networks.

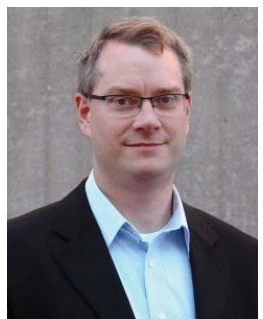

Erik G. Larsson received his $\mathrm{Ph} . \mathrm{D}$. degree from Uppsala University, Sweden, in 2002. Since 2007, he is Professor and Head of the Division for Communication Systems in the Department of Electrical Engineering (ISY) at Linköping University (LiU) in Linköping, Sweden. He has previously been Associate Professor (Docent) at the Royal Institute of Technology (KTH) in Stockholm, Sweden, and Assistant Professor at the University of Florida and the George Washington University, USA.

His main professional interests are within the areas of wireless communications and signal processing. He has published some 80 journal papers on these topics, he is co-author of the textbook Space-Time Block Coding for Wireless Communications (Cambridge Univ. Press, 2003) and he holds 10 patents on wireless technology.

$\mathrm{He}$ is Associate Editor for the IEEE TRANSACTIONS ON COMMUNICATIONS and he has previously been Associate Editor for several other IEEE journals. He is a member of the IEEE Signal Processing Society SAM and SPCOM technical committees. He is active in conference organization, most recently as the Technical Chair of the Asilomar Conference on Signals, Systems and Computers 2012 and Technical Program co-chair of the International Symposium on Turbo Codes and Iterative Information Processing 2012. 